Onkologe 2014 · 20:930-932

DOI 10.1007/s00761-013-2566-1

Online publiziert: 17. August 2014

(c) Springer-Verlag Berlin Heidelberg 2014

\author{
M. Thomas ${ }^{1}$ M. Bamberg ${ }^{2}$ \\ ${ }^{1}$ Abteilung Thoraxonkologie/Innere Medizin, Thoraxklinik, Universität Heidelberg \\ 2 Universitätsklinikum Tübingen
}

\title{
Nichtkleinzellige Lungenkarzinome
}

Bewertung des mediastinalen (N2) und lokoregionären (N1) Lymphknotenstatus. Bisher liegen keine Ergebnisse aus prospektiv-randomisierten Studien zum direkten Vergleich von Resektion und Stereotaxie im Stadium I vor. Flentje et al. vermitteln eine Einschätzung zum aktuellen differenzialtherapeutischen Stellenwert beider Verfahren.

\section{》) Die Prognose dieser Patienten ist nach wie vor nicht günstig}

Bei den erweiterten Resektionen des NSCLC wird von Schirren et al. die Pneumonektomie mit den organerhaltenden Manschettenresektionen verglichen. Selbst in höheren Tumorstadien zeigt die Manschettenresektion ihre Überlegenheit. Funktionelle Einschränkungen nach der Pneumonektomie sind neben der Tumorerkrankung eine weitere schwerwiegende Belastung für den Patienten und zugleich ein Risikofaktor. Zudem lassen sich adjuvante Therapieverfahren nach Organerhalt mit besseren Ergebnissen anwenden, und Arbeitsfähigkeit wie Lebensqualität sind nach einer Manschettenresektion günstiger.

Die Brustwandinfiltration durch ein NSCLC galt Anfang der 1960er Jahre noch als Inoperabelitätskriterium. Welter et al. zeigen auf, dass dieser Eingriff inzwischen im thoraxchirurgischen Alltag zur Routine geworden ist - auch unter Einsatz von größeren Weichteildefekten mit Maßnahmen der plastischen Chirurgie. Beim Pancoast-Tumor sind sowohl Gefäßersatz als auch Wirbelkörperersatz in der oberen Thoraxapertur im onkochirurgischen Konzept etabliert. Ferner zeigen Passlick et al. die etablierten sicheren Resektionen bei Tumorbefall an den großen thorakalen Gefäßen. Insgesamt werden mit den erweiterten Resektionen verbesserte therapeutische Optionen erreicht.

In der metastasierten Tumorsituation kann in der Regel keine Heilung erreicht werden. Jedoch zeichnet sich hier bei der sog. "Oligometastasierung" eine Subgruppe ab, für die, bei entsprechender Therapieführung, vielversprechende Dreijahresüberlebensraten und möglicherweise auch Langzeitüberlebensraten erreicht werden können. Sterzing et al. geben in ihrem Beitrag einen aktuellen Überblick zu den Konzepten der Diagnostik und Therapie beim oligometastasierten NSCLC. Wie eingangs bereits erwähnt wird bei 5 von 6 Patienten mit einem NSCLC keine Heilung erreicht. Letztlich wird bei der Versorgung von Patienten im Stadium IV auch der frühe Blick auf palliativmedizinische Handlungsoptionen notwendig, einmal, um Situationen mit hoher Symptomlast bzw. ausgeprägten Belastungszonen effektiv zu begegnen, aber auch, um in einer angemessenen „End-of-Life-Care“ die Situation für Patienten und Angehörige tragbar zu machen. Eschbach et al. beschreiben in ihrem Beitrag die frühe Integration palliativmedizinischer Handlungsmaßnahmen in den Versorgungskontext beim NSCLC.

Damit decken die Beiträge des aktuellen Hefts von Der Onkologe das Gebiet von modernsten molekularen Therapieansätzen - und der dafür erforderlichen Diagnostik - über moderne Konzepte der lokoregionären Therapie und von aktuellen Ansätzen zum Management der oligometastatischen Erkrankung bis hin zum modernen Führungs- und Steuerungs- 
Hier steht eine Anzeige.

黛 Springer 
konzept bei einer nicht heilbaren metastasierten Krebserkrankung gut ab.

Für die Schriftleiter des Schwerpunktthemas

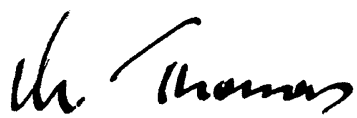

M. Thomas

Für die Herausgeber<smiles>CCCCC</smiles>

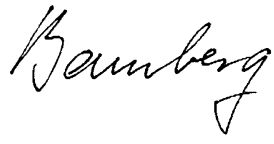

M. Bamberg

\section{Korrespondenzadresse}

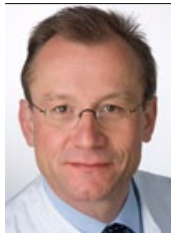

Prof. Dr. M. Thomas

Abteilung Thoraxonkologie/ Innere Medizin, Thoraxklinik, Universität Heidelberg

Amalienstraße 5,

69126 Heidelberg

michael.thomas@

med.uni-heidelberg.de

\section{Einhaltung der ethischen Richtlinien}

Interessenkonflikt. M. Thomas und M. Bamberg geben an, dass kein Interessenkonflikt besteht.

\section{Förderpreis für Konzepte zur Verbesserung der intensiv- medizinischen Versorgungs- qualität 2015}

Der vom Wissenschaftlichen Verein zur Förderung der klinisch angewendeten Forschung in der Intensivmedizin e.V. (WIVIM) ausgeschriebene Förderpreis in Höhe von $€ 3.000$ wird für innovative Projekte und Projektvorhaben vergeben, die auf eine nachhaltige Verbesserung der intensivmedizinischen und intensivpflegerischen Versorgungsqualität abzielen. Hierbei werden vorzugsweise Projekte berücksichtigt, die anerkannte Instrumente des Qualitätsmanagements (PDCA-Zyklen) verwenden.

Der Förderpreis wird jährlich ausgeschrieben und vergeben. Um den Förderpreis können sich alle Teilnehmer des Symposiums Intensivmedizin + Intensivpflege bewerben. Berücksichtigt werden Projekt-Manuskripte in deutscher oder englischer Sprache, die bis zum 15. Dezember 2014 in elektronischer Form als PDF an kontakt@wivim.de eingereicht worden sind.

Für die Bewerbung sind ein formloses Anschreiben und eine kurze strukturierte Projektbeschreibung notwendig. Die Projektbeschreibung sollte neben der Thematik insbesondere das zugrunde liegende Konzept und die Zielstellung, die methodische Aufarbeitung, Eckpunkte der Durchführung und der anschließenden Evaluierung sowie Informationen zur Gesamtlaufzeit des Projektes enthalten.

Im Fall der Preisvergabe soll der Preisträger seine Ergebnisse, ggf. auch in Form eines vorläufigen Projektberichts, auf dem im darauffolgenden Jahr stattfindenden Symposium für Intensivmedizin + Intensivpflege präsentieren.

Die Preisvergabe nimmt die Jury während des Bremer Symposiums für Intensivmedizin + Intensivpflege 2015 vor.

Rückfragen bitte an:

Herrn Prof. Dr. Herwig Gerlach,

E-Mail: herwig.gerlach@vivantes.de

\section{DKG-Internetportal bietet News-Service für Onkologen}

In kaum einem medizinischen Fachgebiet schreitet die Forschung so schnell voran wie beim Thema Krebs. Selbst für manchen Mediziner ist es zuweilen schwierig, mit dem Taktschlag der neusten Erkenntnisse Schritt zu halten. Tagesaktuell auf dem Laufenden bleiben interessierte Ärzte mit den Nachrichten für Fachpublikum auf dem Internetporta der Deutschen Krebsgesellschaft e. V. (DKG). Unter www.krebsgesellschaft.de/news_arzt gibt das Portal Einblick in sein Nachrichtenarchiv, das über eine Schlagwortsuche zu Recherchezwecken verfügt.

Bei Bedarf liefert eine Onkologie-App für das iPhone zusätzlich und zuverlässig täglich aktualisierte Meldungen aus dem interdisziplinären Fachbereich der Onkologie, bereichert durch Berichte von internationalen und nationalen Kongressen sowie News aus Gesundheitspolitik und -wirtschaft. Die Onkologie-News-App ist eine Kooperation des DKG-Internetportals mit Springer Medizin. Die Inhalte basieren auf dem Webangebot www. krebsgesellschaft.de und dem Arzt-Portal www.springermedizin.de. Informationen zur Nutzung finden Ärzte unter www.krebsgesellschaft.de/onko_app.

Quelle:Deutsche Krebsgesellschaft e.V.(DKG), www.krebsgesellschaft.de 